\title{
Full System for Translational Studies of Personalized Medicine with Free-Moving Mice
}

\author{
Sandro Carrara*, Camilla Baj-Rossi, Sara Seyedeh Ghoreishizadeh,Stefano Riario, \\ Grégoire Surrel, Francesca Stradolini, Cristina Boero, Giovanni De Micheli, Enver G. Kilinc, Catherine Dehollain \\ Swiss Federal Institute of Technology (EPFL), Lausanne, Switzerland \\ *sandro.carrara@epfl.ch
}

\begin{abstract}
A full remotely powered system for metabolism monitoring of free-moving mice is presented here. The fully implantable sensing platform hosts two ASICs, one off-the-shelf micro-controller, four biosensors, two other sensors, a coil to receive power, and an antenna to transmit data. Proper enzymes ensure specificity for animal metabolites while Multi-Walled Carbon Nanotubes ensure the due sensitivity. The remote powering is indeed provided by inductive coils located under the floor of the mouse' cage. Two different approaches where investigated to ensure freedom of movement to the animal. The application to studies for personalized medicine is demonstrated by showing continuous monitoring of both glucose and paracetamol.
\end{abstract}

\section{INTRODUCTION}

Translational medicine is an extremely effective approach to progress in diseases characterization as well as in identifying and optimizing new therapeutic treatment [1]. The use of small animal, like mice, is recommend to save time and money. The extremely reduced sizes of a mouse (only $5 \mathrm{~cm}$ in length) pose technological challenges in developing extremely tiny and highly integrated fully implantable chip to monitor its metabolism. The further request to allow freemovements to the mouse as well as to avoid animal stress impose further challenges on the system powering. The size of the implant needs to be small and lightweight for the comfort of the animal. Therefore, the implant should be batteryless. The magnetic field is one of the most well known solutions for remote powering [2]. The power is delivered to an implant by using a magnetically coupled coil pair. This study presents a fully implantable device for remote monitoring of mice metabolism in study related to personalized medicine by investigating two different powering approaches to allow free movement to the animal.

\section{THE Fully IMPLANTABLE DEVICE}

\section{A. The Device Architecture}

The whole fully implantable device is build out of three main different sub-units (Fig. 1): (i) a passive chip realized on silicon substrate that hosts four different biosensors plus one temperature (T) and a $\mathrm{pH}$ sensor; (ii) an intermediate layer realized on PCB (Printed Circuit Board) that hosts two dedicated ASIC ICs and an off-the-shelf microcontroller; (iii) one coil at the bottom that behave as receiving antenna to power the whole device.
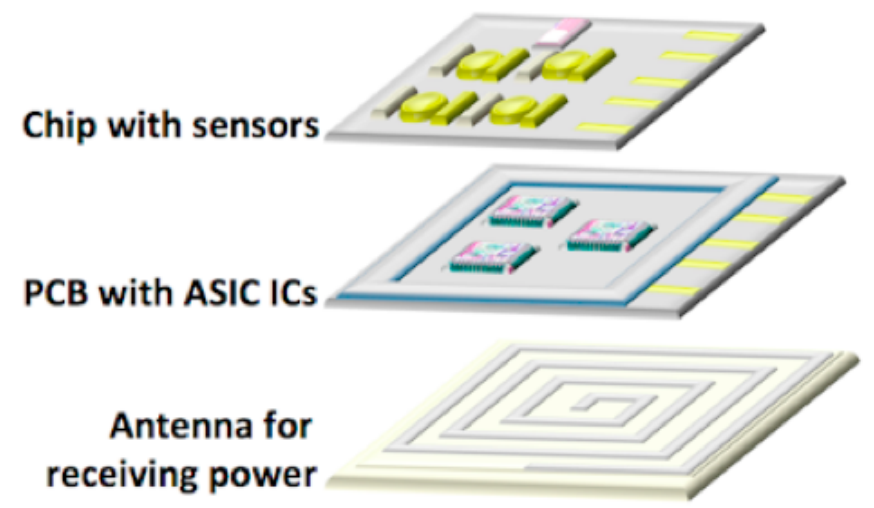

Fig. 1. The system architecture of the implantable device

\section{B. The Fabrication of the Passive Chip}

The micro fabrication of the passive chip was realized with a two-masks process flow. Silicon wafers with $500 \mathrm{~nm}$ of native oxide were chosen as substrate. Chip metallization was realized by evaporation of $10 \mathrm{~nm}$ of Ti, followed by $100 \mathrm{~nm}$ of Pt. Metal passivation was made via atomic layer deposition of $\mathrm{Al}_{2} \mathrm{O}_{3}$ followed by dry etching with Argon Ion Milling. Details on the process flow can be found in [3]. The platform measures $12 \times 11 \mathrm{~mm}$ in order to fit the size of the coil $(12 \times 12$ $\mathrm{mm}$ ) and the wire bonding. The platform hosts an array of four independent cells in the three-electrode configuration. All electrodes are made in $\mathrm{Pt}$

\section{The Functionalization of the Biosensors}

For the glucose measurement, the working electrodes were nanostructured with $0.3 \mu \mathrm{g}$ MWCNTs by drop cast, followed by the drop cast of $2 \mu \mathrm{l}$ of a glucose oxidase solution (15 $\mathrm{mg} / \mathrm{ml})$, as reported in [3]. For the drug measurements (Paracetamol), the electrodes were used without modifications. 


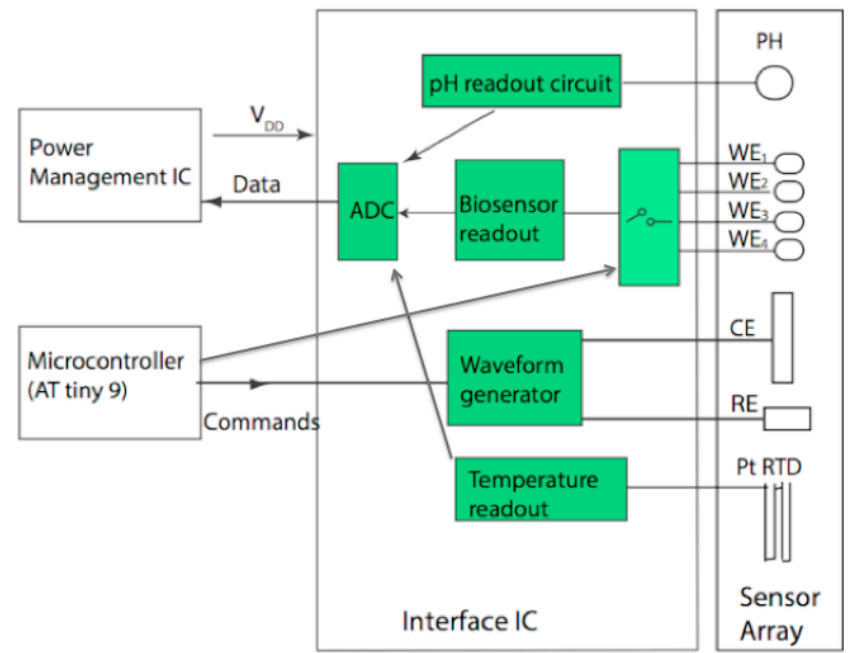

Fig. 2. The architecture of the frontend IC

\section{THE ASIC ICS}

\section{A. The Sensors Frontend}

A mixed-mode IC is implemented to interface the sensor array. The block diagram of the interface IC (IC) is shown in Fig. 2, together with its connections with the sensor and other devices on the platform. The IC controls and reads out four bio-molecular sensors, as well as a $\mathrm{pH}$ sensor and a temperature sensor for calibration. The IC supports both cyclic voltammetry (CA) and chronoamperometry (CV). Different voltage profiles are generated to control CV and CA by using a single fully on-chip waveform generator. The IC reads out the electrochemical sensors and streams out the digitized measured data. The streamed out data is given to the other IC to be transmitted. The waveform generator is commanded from a standard interface. The required commands are provided by a tiny commercial microcontroller. The IC is implemented in 0.18 um technology and consumes $0.93 \mathrm{~mW}$ from $1.8 \mathrm{~V}$ supply voltage. Electrical measurements show that the readout current range is $1650 \mathrm{nA}$ with 8-bit resolution. More details on the design and its testing are reported in [4].

\section{B. The Energy Manager}

On-chip implantable solution is composed of power management circuits. The induced $\mathrm{AC}$ voltage on the implant coil is converted to a DC voltage by a passive rectifier and a clean and stable $1.8 \mathrm{~V}$ supply voltage is created by a low drop-out voltage regulator [5].

\section{THE PACKAGING OF THE IMPLANT}

\section{A. The Sensors Frontend}

The epoxy adhesive (EP42HT-2Med system) was used to assembly the PCB with the antenna and the electronic component, with the sensing platform. The interconnections between the platinum pads of the passive chip and the gold pads on the PCB were realized with Al wire bonding and were protected with a glob top protection of $0.3 \mathrm{~mm}$.

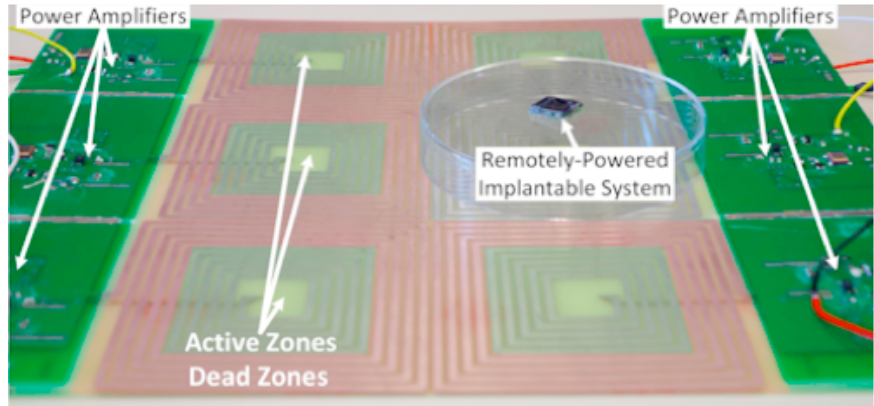

Fig. 3. Coil array powering system

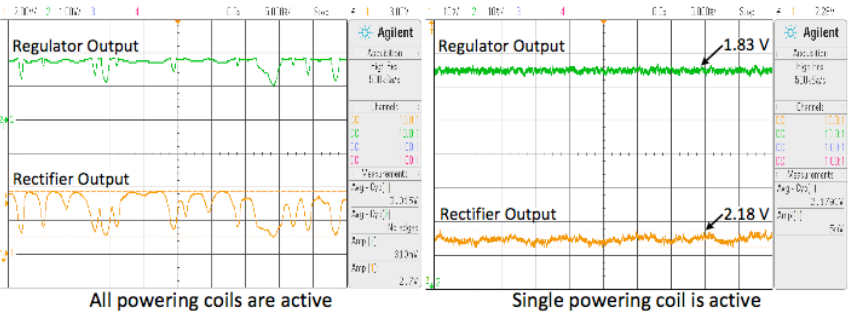

Fig. 4. Drawback of the coil array powering solution

Two subsequent $5 \mu \mathrm{m}$ layers of Parylene $\mathrm{C}$ were deposited by chemical vapor deposition using a Comelec C-30-S Parylene Deposition System. Parylene C was used to cover the whole device but not the electrode array that needs to be in contact with fluids. A biocompatible Silicone (NuSil MED-6233), was used to cover the edges and to fill the spaces between the platform and the PCB. Finally, the electrodes were functionalized for the electrochemical measurements as previously described.

\section{THE REMOTE POWERING Systems}

\section{A. Coil Array}

The implant requires continue power for any position of the animal in the cage meanwhile the animal must move freely inside. Accordingly, a coil array is implemented to cover all the bottom of the cage and transfer the power to the implant. .3 shows the approach with the coil array. The powering coils are driven by class-E power amplifiers at $13.56 \mathrm{MHz}$ frequency. Each power amplifier is optimized for maximum power transfer efficiency [6]. The power transfer efficiency decreases when the implantable system moves from the center to the edges of the powering coil. Therefore, the transferred powered needs to be increased to keep the implantable system active. However, after a certain distance from center, the remote powering signal creates significant noise on the communication band due to the increased transfer power. Accordingly, there are some dead zones where the transfer efficiency decreases drastically and also the communication becomes crucial as shown in Fig. 3. In addition, the powering coils create large noise on the remote powering signal when all the coils are active as shown in Fig. 4. This noise can be suppressed by adding extra capacitors to the output of the rectifier [5]. However, the digital control block of the implantable system demands abrupt supply voltage to initialize properly. 


\section{B. Servo-Controlled Moving system}

In this second approach, we propose a servo-controlled remote powering system that locates the animal in the cage and delivers the power to the implantable system efficiently [7]. Fig. 5 shows the servo-controlled remote powering system of the implantable system. The system has two $\mathrm{X}$ and $\mathrm{Y}$ axes rails to move the powering coil under the cage according to the position of the animal in the cage. In order to define the animal position, a permanent magnet is tracked and monitored by the magnetic field sensors. The powering coil is driven by an efficient class-E type power amplifier that is optimized at $13.56 \mathrm{MHz}$. The servo-controlled system helps to keep the powering coil position aligned to the implantable system to efficiently transfer the power, which decreases the noise of the power amplifier on the communication frequency band. Therefore, a reliable data transmission is achieved. The aforementioned noise due to using multiple coil for remote powering is also suppressed since single powering coil is used to the power transfer as shown in Fig. 3.

\section{The REMOTE MONITORING System}

\section{A. The Architecture for Receiving Data}

The implantable platform has onboard a frontend chip [4] that provides the $8 / 10$ bits coded information of the current signal flowing inside the biosensors as a digital word of 20 bits. This signal drives an $869 \mathrm{MHz}$ OOK transmitter [6], which modulates this information in order to be processed by the external reader. The external reader is composed of three main parts compose the last one: the demodulator, the Bluetooth transmitter and the Android interface

\section{B. The Demodulator}

An custom designed antenna receives the modulated signal from the implantable chip with the tuned radio frequency receiver. The output $\left(\mathrm{V}_{\mathrm{f}}\right)$ is sent to a former project board [6], which is a tuned RF receiver. The receiver is able to clean the signal and give to it the same waveform of the original one provided by the chip inside the implantable platform due to the logarithmic amplifier and a high-speed comparator (Fig. 6).

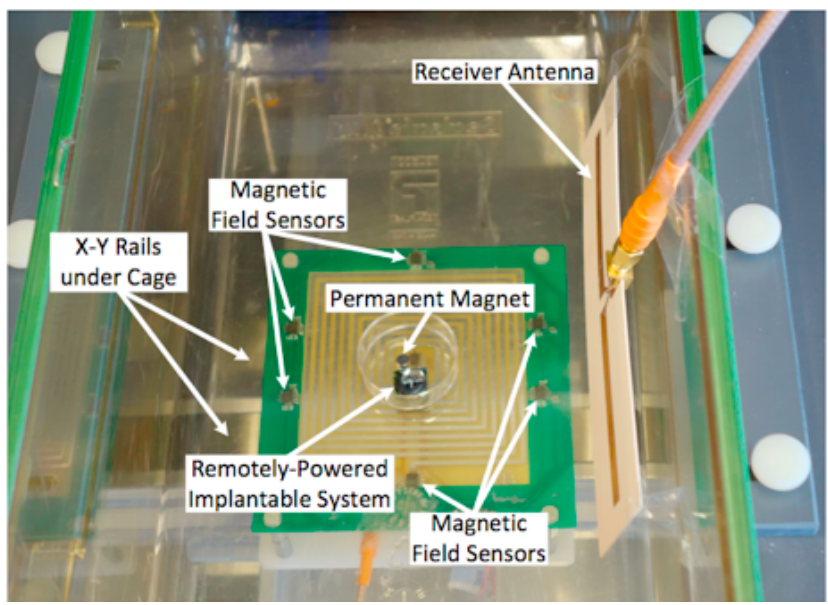

Fig. 5. Servo-controlled remote powering system

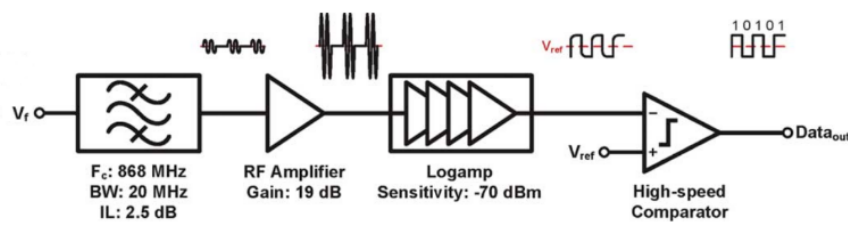

Fig. 6. Demodulator [6]

\section{Bluetooth Transmitter}

The cleaned digital signal is sent to another board which is in charge of analyze, validate and transmit via Bluetooth the data to the Android interface. The on-board microcontroller is able to sample each bit of the digital signal (Data ${ }_{\text {out }}$ ), decode it and understand if a transmission error has occurred. When the data has been validated, is sent by the well-known UART protocol to a commercial Bluetooth module as a word of two bytes.

\section{Android Interface}

For a continuous monitoring of the concentration of the substances we want to measure, an android application has been developed starting from a previous work [8]. The most important improvement is the possibility to zoom the curves and to filter the noise with many filtering techniques (moving average, median value, IIR filter, ecc...) while the interface is acquiring and plot the data in time. Reliability and data space occupation has been optimized in order to let the user save a significant number of curves according to the big external flash memory of the device, which can be any tablet or smartphone.

\section{SYSTEM IN-VITRO TESTS}

\section{A. Electrochemical Measurements}

Electrodes were tested for glucose or Paracetamol sensitivity with chronoamperometry at $+650 \mathrm{mV}$. The sensors were covered with $200 \mu \mathrm{l}$ of a $100 \mathrm{mM}$ phosphate buffered saline (PBS) solution ( $\mathrm{pH} 7.4)$, and then tested against solutions at increasing concentration of glucose or Paracetamol.

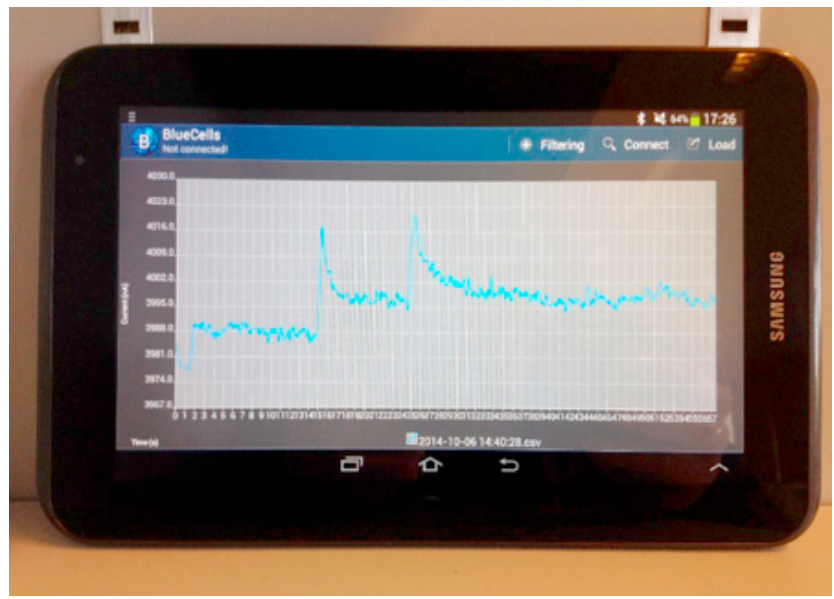

Fig. 7. Chronoamperometry on the Android interface 


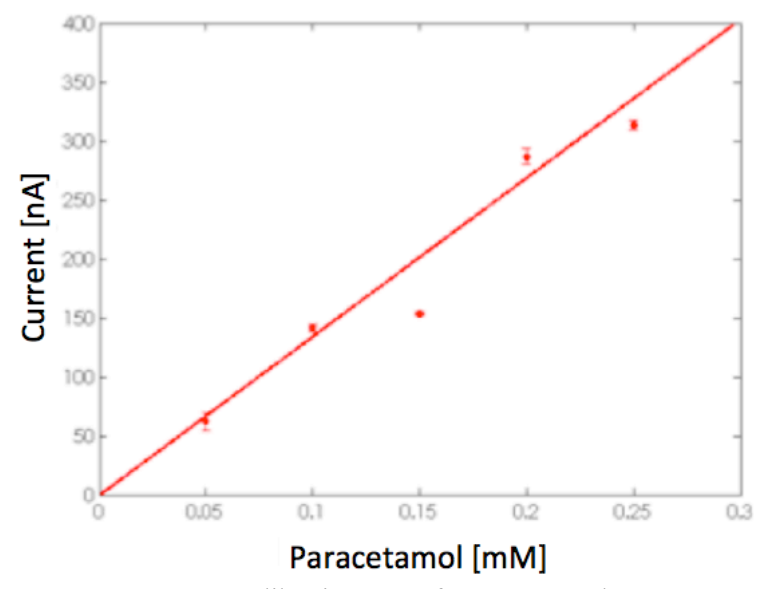

Fig. 8. Calibration curve for paracetamol

Sensitivity and limit of detection (LOD) were evaluated after linear regression of the data, according to [9]. Measurements with glucose and Paracetamol were carried out with both the fixed coil and the intelligent moving remote powering systems. Here we report the calibration obtained with the moving powering system. To perform a calibration, the chip was kept at a fixed position and the chip was covered with $200 \mu \mathrm{l}$ of PBS at increasing concentrations of glucose or Paracetamol. Calibration lines (one example reported in Fig. 8 ) are calculated from the evaluation of the current steps, by measuring the difference between the reached current value and the baseline (corresponding to the current at $0 \mu \mathrm{M}$ of glucose or Paracetamol). For glucose we obtained a sensitivity of $1.3 \pm 0.4 \mathrm{nA} / \mathrm{mMmm}^{2}$ and a LOD of $0.2 \pm 0.1 \mu \mathrm{M}$, which is relevant in many clinical applications. Many other metabolites of clinical interest (e.g. lactate, glutamate) can be monitored with our sensing platform, by changing the enzyme that is employed for the electrode functionalization.

The anti-inflammatory drugs Paracetamol was monitored with the same system. We obtained a sensitivity of $1.7 \pm 0.5$ $\mathrm{nA} / \mathrm{mMmm}^{2}$ and a LOD of $12 \pm 9 \mu \mathrm{M}$, which is compatible with the physiological range for Paracetamol [10].

\section{B. Data Acquisition with the Moving System}

We also performed measurements where the chip was moved in the cage in order to test the capability of the intelligent powering system to track the chip position, to transfer the power and the ability of the receiving system to acquire the data and showing them on the tablet. The data stream in Fig. 9 clearly shows that during the displacement no data are transmitted.

\section{CONCLUSIONS}

We investigated two approaches for the remote powering of a fully implantable multi-panel device for translational medicine. The first approach proposes a series of coils powering the implant once the mouse is located on top of them. The second approach presents a single coil that follows the mouse thanks to a magnet located in the implant and a robot that moves the coil. The multi-coils system presents limitations dealing with "dead zones" of powering in the cage while the moving-coil ensures better continuity on powering to the implant thanks to the good performance of the feedback and actuation system.

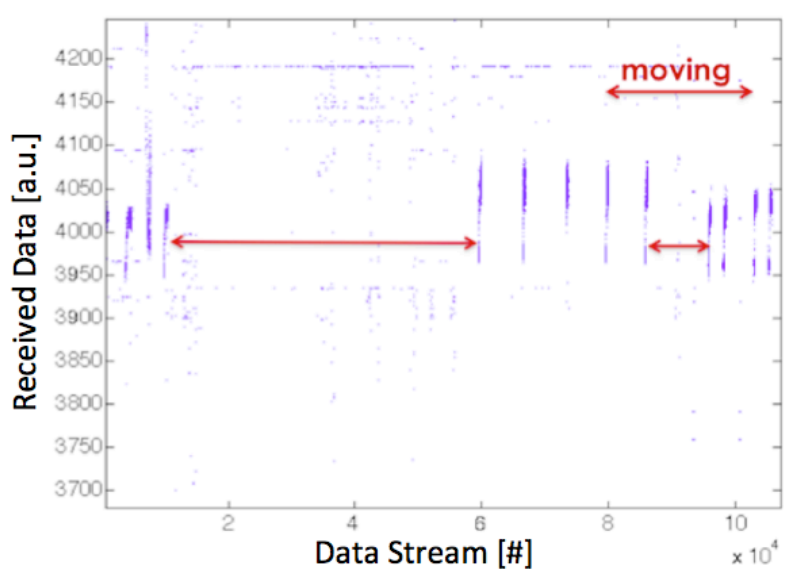

Fig. 9. Data stream during chip displacement in the cage

By using this second approach, the continuous monitoring of both a disease marker (glucose) and a therapeutic compound (paracetamol) was demonstrated.

\section{Acknowledgments}

This research has been supported by the Swiss SNF projects Sinergia CRSII2_147694 / 1 entitled: "Innovative Enabling Micro-Nano-Bio-technologies for Implantable systems in molecular medicine and personalised therapy - project prolongation"

\section{References}

[1] Jucker M., The benefits and limitations of animal models for translational research in neurodegenerative diseases, Nature Medicine 16(2010) 1210-1214

[2] Peng Cong, Nattapon Chaimanonart, Wen H. Ko, and Darrin J. Young, A Wireless and Batteryless 10-Bit Implantable Blood Pressure Sensing Microsystem With Adaptive RF Powering for Real-Time Laboratory Mice Monitoring, IEEE J. Solid-Sate Circuits, 44(2009) 3631-3644L.

[3] C. Baj-Rossi, E. G. Kilinc, S. S. Ghoreishizadeh, D. Casarino, T. Rezzonico Jost, C. Dehollain, F. Grassi, L. Pastorino, G. De Micheli and S. Carrara, "Full Fabrication and Packaging of an Implantable Multi-panel Device for Monitoring of Metabolites in Small Animals", IEEE Transaction on Biomedical Circuits and Systems 8(2014) 636647.

[4] Sara S. Ghoreishizadeh, Camilla Baj-Rossi, Andrea Cavallini, Sandro Carrara, Giovanni De Micheli "An Integrated Control and Readout Circuit for Implantable Multi-Target Electrochemical Biosensing," IEEE Transactions on Biomedical Circuits and Systems, 8(2014) 891898

[5] E.G. Kilinc, et. al., "Remotely powered telemetry system with dynamic power-adaptation for freely moving animals," in IEEE BioCAS 2012, 260-263

[6] E.G. Kilinc, et. al., "A remotely powered implantable biomedical system with location detector," in Biomedical Circuits and Systems, IEEE Transactions on Biomedical Circuits and Systems, 9(2015).113-123

[7] E.G. Kilinc, et. al., "A system for wireless power transfer of microsystems in-vivo implantable in freely moving animals," in Sensors Journal, IEEE, 14(2014) 522-531

[8] Jacopo Olivo, Lorenzo Foglia, Maria Antonietta Casulli, Cristina Boero, Sandro Carrara, and Giovanni De Micheli, "Glucose and Lactate Monitoring in Cell Cultures with a Wireless Android Interface”, IEEE BioCAS 2014, 400-403

[9] J. Mocak, A. Bond, S. Mitchell, and G. Scollary, "A statistical overview of standard (iupac and acs) and new procedures for determining the limits of detection and quantification: Application to voltammetric and stripping techniques," Pure and Applied Chemistry,. 69(1997) 297-328

[10] Rawlins, M. D., D. B. Henderson, and A. R. Hijab. "Pharmacokinetics of paracetamol (acetaminophen) after intravenous and oral administration". European journal of clinical pharmacology 11(1977) 283-286. 